\title{
Relational Gestalt approach to building a COVID-19 community of practice in a hospital setting
}

\author{
Justin T Denholm, ${ }^{1,2}$ Douglas Johnson, ${ }^{1,2}$ Gina C Denholm, ${ }^{3}$ Eva Deligiannis ${ }^{3}$
}

${ }^{1}$ Victorian Infectious Diseases Service, The Royal Melbourne Hospital, Parkville, Victoria, Australia

${ }^{2}$ Department of Infectious Diseases, The Peter Doherty Institute for Infection and Immunity, Melbourne, Victoria, Australia

${ }^{3}$ Gestalt Therapy Australia, Carlton North, Victoria, Australia

Correspondence to Professor Justin T Denholm, The Royal Melbourne Hospital, Parkville, Victoria, Australia; justin.denholm@mh.org.au

Received 21 December 2021 Accepted 23 January 2022

\section{ABSTRACT}

Background The COVID-19 pandemic has been highly disruptive in many work environments, particularly those related to direct provision of healthcare. Significant organisational change has been required at many levels, with attendant risks of both impaired service delivery and psychological impact on staff. Relational organisational Gestalt (ROG) is an approach that emphasises interpersonal connection about shared reality, which can be used in a variety of ways to support organisational change.

Methods We established a community of practice in an acute hospital setting using ROG approaches during a COVID-19 pandemic wave. This group primarily consisted of senior medical staff redeployed to COVID-19 ward duties, who met daily for facilitated sessions centred around inpatient activities.

Results Emerging group practices and outputs are described, including adjustments to group processes in response to situational need. A ROG approach was perceived as both effective in supporting rapid change in practice, and for providing psychological support for staff members.

Conclusions ROG can be a useful and adaptive model for supporting staff and systems through times of change. Further exploration and evaluation of this approach in a variety of healthcare environments and applications will be valuable.

\section{INTRODUCTION}

The Royal Melbourne Hospital is a large public quaternary referral centre, with over 11000 staff and 1400 inpatient beds. The infectious diseases service, and its joint venture the Peter Doherty Institute for Infection and Immunity, also provides statewide, national and international services through the Victorian Tuberculosis Program, WHO Collaborating Centres for Viral Hepatitis and Influenza, and dedicated clinical response services for outbreak and pandemic infections. From January 2020, the Royal Melbourne Hospital has also been a 'streaming' hospital for COVID-19, including provision of inpatient and outpatient services, vaccination hubs and programmatic support for the Victorian Department of Health.

The Victorian COVID-19 experience in 20202021 has been characterised by distinct waves of infection. Particularly during these periods, healthcare services have been disrupted for reasons including closure to elective medical and surgical procedures, redistribution of staff, beds and resources to COVID-19 management, and both direct and indirect impact on staff across the sector. ${ }^{12}$ Times of major change such as this are increasingly described as volatile, uncertain, complex, ambiguous (VUCA) environments, where pre-existing leadership and organisational structures may be less effective. ${ }^{3}$ It is critical to explore and evaluate alternative approaches to organisational management, and consider how changing service models may occur in ways which are both effective and empowering for healthcare workers. This article describes the implementation of a major COVID-19 service change, supported by a relational organisational Gestalt (ROG) approach.

\section{RELATIONAL ORGANISATIONAL GESTALT}

ROG is an adaptive method based on dialogue and shared reflection. ${ }^{4}$ The model is derived from Gestalt psychotherapy, and has been developed for use in change management within a range of contexts. While traditional models of organisational change have frequently used 'top-down' approaches for communicating a need for change and its implementation, ROG employs an 'ecological' approach in which solutions and priorities emerge from interpersonal reflection. ${ }^{5}$

Key to ROG practice is providing opportunities for groups to reflect together about their experience and environment. Groups operating with a ROG framework will frequently make use of an 'self, other, situation' model, which draws participants' attention ('ethical presence') to their shared situational reality as a basis for emerging reflection and action (figure 1). ${ }^{6}$ Its strengths in a healthcare environment include adaptability to new situations, and an approach that puts relationships at the centre of change management. ${ }^{7}$ This provides opportunities for effective and lasting change, and mutual psychological support in difficult environments.

\section{INTERVENTION}

During the initial pandemic surge, rapid expansion of services relating to inpatient care for COVID-19 occurred. In order to reduce the risk of transmission and increase bed availability, a progressively larger number of wards were converted to provide COVID-19 services. These wards, previously providing for areas such as general medicine, stroke and postsurgical recovery, were staffed with medical, nursing and allied health workers previously providing these services, with movement between wards avoided to reduce further transmission within the hospital. These arrangements meant that, over a relatively brief period of several weeks, medical management was primarily provided by doctors who had not worked in COVID-19-specific or infectious disease practice previously. 


\section{CONTEXT}

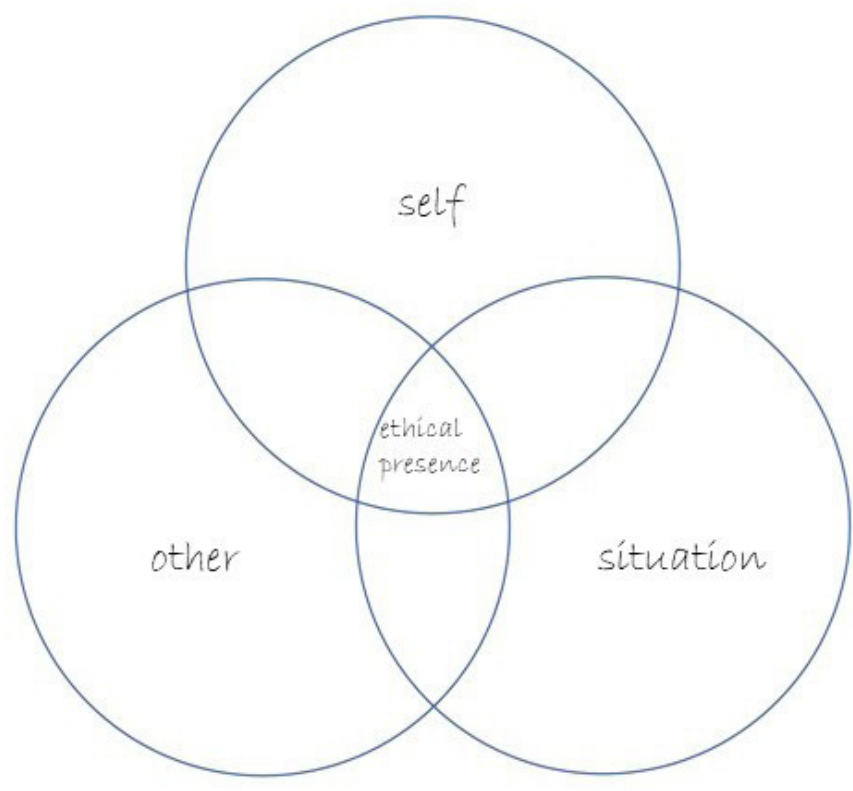

CULTURE

Figure 1 The SOS model. Adapted from Chidiac and Gestalt. ${ }^{5}$ SOS, self, other, situation.

In addition to providing written guidelines, specialty referral services and other clinical support tools, we wanted to establish a structure for supporting medical staff transitioning into these new roles, and wondered whether the principles of relational Gestalt could inform this practice.

We determined that the key features for the group at formation were that it should be voluntary, aim to be informal with limited hierarchy, be non-judgemental and psychologically safe, and be humble and recognise mutual expertise. Structurally, this was reflected by establishing a daily videoconference which was made available to all medical staff who wished to participate. This was particularly offered to those working on COVID-19 wards, but not limited to this group, and our experience was that individuals anticipating future involvement also began to attend.

A facilitator for each session was designated, among a small group of clinicians with previous COVID-19 management experience and resonance with ROG approaches. Establishment of this group was received positively, and over the first 3 weeks of operation, typical attendance grew from 5-6 people to 20-25 daily. With increasing numbers also came a greater diversity of professional backgrounds, including consultants in palliative care, anaesthetics, cardiac surgery, nephrology, rheumatology and neurology. This breadth allowed facilitators to consciously draw on the range of expertise represented, working on the principle of exchanging their own role as 'expert' in order to build stronger collaboration and inclusiveness. ${ }^{8}$

While no formal agenda existed, as the group progressed an emergent approach developed with periodic reflection and modification. The facilitator would typically:

1. Arrive early to the scheduled meeting and have informal discussion about topics other than COVID-19 while others arrived.
2. Invite each ward team in turn to share about their morning, particularly to highlight a question that had arisen, something they had learnt, a barrier to care or an area of uncertainty they had experienced. Inviting experiences from more junior staff present was prioritised first. Facilitators modelled sharing their own ward experiences broadly, including open disclosure of uncertainty and emotional responses to situations.

3. Encourage group discussion on emergent themes from the ward reports. Facilitators would provide some expert input where appropriate, but deliberately sought to preference inviting commentary from a range of other participants, with emphasis on gender, racial and discipline diversity in comment.

4. Conclude meetings on time, with comment on a positive element. This varied from reflecting on something the group had learnt together, an expression of gratitude to participants, or feedback on a good outcome such as a patient who had recovered or returned to thank the hospital.

In parallel to these meetings, the community of practice developed a number of resources, especially to support new members as they joined. These included lists of frequently asked questions, and guidelines developed in response to new treatments or published data. Commonly, individuals or small groups would be tasked to develop materials 'out of session' and return to the wider group for feedback, allowing real-time development in response to need.

The group continued to meet daily through SeptemberOctober 2021. As vaccination rates increased, hospital admissions decreased through November, and from a peak of 8 dedicated COVID-19 wards, one remained open by early December. Group members discussed optimal approaches through this period, leading to a decrease in meeting frequency and eventual conclusion after a 3 -month period of operation.

\section{SUMMARY AND LESSONS LEARNT}

In our experience, ROG was a useful framework for supporting groups through significant change, particularly in a VUCA environment. Initially, there were concerns about whether a daily meeting of this type might impair efficiency, both due to the frequency of meeting and the unstructured approach used in our ROG model. However, our experience was that the group process improved efficiency, by leading quickly to establishing agreed approaches to common scenarios, meaning that new members were able to quickly harmonise with best practice.

Several characteristics of our process meant that ROG was a particularly suitable approach. Group members were experienced professionals in often unrelated fields, and a psychologically safe environment allowed acknowledgement of uncertainty and a need for help. This also allowed modelling of collaborative and supportive engagement with uncertainty for more junior staff. Mutual vulnerability enabled recognition that the expertise of each member was valuable, and contributed to support of other group members. Our experience was that the process functioned best with a moderately sized (10-25) group of people, especially when a diverse set of skills and backgrounds was represented. We observed that towards the end of the process, when hospital admissions fell and group size declined, the use of alternative strategies, such as 1-on-1 consultation support, re-emerged.

As ROG groups risk being unstructured in nature, active facilitation facilitation of the group and regular feedback was essential to support meaningful outcomes. The lead facilitator was an infectious diseases physician with training in ROG, 
who separately participated in peer-support for ROG practitioners. External support allowed for critical reflection on group dynamics and opportunities to adjust practice in real-time. These supports were valuable for implementation, although we also found that other group members were intuitively responsive to the ROG approach and able to facilitate sessions on an ad hoc basis.

Key to the group's success was formation around a set of genuine perceived needs for members. While participation was voluntary, attendance during peak activity generally exceeded the number of staff rostered for COVID-19 duties, highlighting an effective response. We consider that these felt needs included assistance in unfamiliar medical decision-making, relational connection and psychological support in an environment otherwise orientated towards physical isolation and efficiency in healthcare delivery. Cessation of the group when no longer needed was equally important as a reflection of its purpose, rather than a structure to be established and maintained for its own sake.

In summary, ROG provided helpful insights into developing a supportive group process in the context of COVID-related change, perceived as both useful for harmonising practice and providing psychological support. Within our own institution, we intend to further develop this model into other areas of work, particularly those involving shared decision making in areas of diagnostic and therapeutic uncertainty. Further exploration and evaluation of ROG in healthcare environments will be valuable.

Twitter Justin T Denholm @justin_denholm

Acknowledgements The authors are grateful for support from the Royal Melbourne Hospital and participation from staff in this community of practice, and to members of a Relational Organisational Gestalt peer support group for feedback during its operation.
Contributors All authors were involved in this intervention, and in development and preparation of this manuscript. Initial manuscript drafts were prepared by JD, with all authors approving final manuscript prior to submission.

Funding The authors have not declared a specific grant for this research from any funding agency in the public, commercial or not-for-profit sectors.

Competing interests None declared.

Patient consent for publication Not applicable.

Ethics approval This study does not involve human participants.

Provenance and peer review Not commissioned; externally peer reviewed.

This article is made freely available for personal use in accordance with BMJ's website terms and conditions for the duration of the covid-19 pandemic or until otherwise determined by BMJ. You may use, download and print the article for any lawful, non-commercial purpose (including text and data mining) provided that all copyright notices and trade marks are retained.

\section{REFERENCES}

1 Chow EPF, Hocking JS, Ong JJ, et al. Sexually transmitted infection diagnoses and access to a sexual health service before and after the National lockdown for COVID-19 in Melbourne, Australia. Open Forum Infect Dis 2021;8:ofaa536.

2 Migliori GB, Thong PM, Alffenaar J-W, et al. Gauging the impact of the COVID-19 pandemic on tuberculosis services: a global study. Eur Respir J 2021;58:2101786.

3 Murugan S, Rajavel S, Aggarwal AK. Volatility, uncertainty, complexity and ambiguity (VUCA) in context of the COVID-19 pandemic: challenges and way forward. Int J Health Syst Implement Res 2020;4:10-16.

4 Chidiac Marie-Anne, Chidiac MA. An organisational change approach based on gestalt psychotherapy theory and practice. J Organization Change Management 2013;26:458-74.

5 Chidiac MA, Gestalt D-VS. The good and the concept of ethical presence. Brit Gestalt J 2020;29:21-9.

6 Denham-Vaughan S, Chidiac Marie-Anne, Chidiac MA. Sos: a relational orientation towards social inclusion. Mental Health Soc Inclusion 2013:17:100-7.

7 Clark M, Denham-Vaughan S, Chidiac M-A. A relational perspective on public sector leadership and management. Int J Leadership Pub Services 2014;10:4-16.

8 Fairfield M. The relational movement. Brit Gestalt J 2013;22:22-35. 